\title{
Special issue on computational techniques and applications
}

\author{
Georges Zaccour
}

Received: 9 May 2014 / Accepted: 12 May 2014 / Published online: 4 June 2014

(C) Springer-Verlag Berlin Heidelberg 2014

This issue is based on presentations at the tenth International Conference on Computational Management Science held at HEC Montréal, Canada. The topics covered highlight the role played by computational techniques to solve relevant problems in management science.

The CMS conference is an annual meeting associated with the Journal of Computational Management Science. The aim of this conference is to provide a forum for theoreticians and practitioners from academia and industry to exchange knowledge, ideas and results in a broad range of topics relevant to the theory and practice of computational methods, models and empirical analysis for decision making in economics, engineering, finance and management. The focus is on all computational aspects of management science: theoretical and empirical studies of computational methods, models and empirical analysis. These include computational economics, finance and statistics, energy, scheduling, supply chains, design, analysis and applications of optimization algorithms, deterministic, dynamic, stochastic, robust and combinatorial optimization models, solution algorithms, learning and forecasting such as neural networks and genetic algorithms, models and tools of knowledge acquisition, such as data mining, and all other topics in management science with the emphasis on computational paradigms. The ten papers in this special issue reflect this diversity.

The first two papers deal with the computation of equilibria in games. Savani and von Stengel present the "Game Theory Explorer", a software tool to create and analyze games as in extensive and strategic forms. This software uses state-of-the-art algorithms to compute all Nash equilibria of a game. The authors also show that in a tutorial fashion how the program is used, and the ideas behind its main algorithms. Finally, they report on experiences with the architecture of the software and its

G. Zaccour $(\varangle)$

GERAD, HEC Montréal, Montreal, Canada

e-mail: georges.zaccour@gerad.ca 
development as an open-source project. Schimit, Santos and Soares use probabilistic cellular automata to model a population in which the cells represent individuals that interact with their neighbors playing a game, of the Prisoner's Dilemma or Hawk-Dove variety. Their main purpose is to test different fitness functions for evaluating the generation of new individuals, which will have characters of the best adapted individuals in a neighborhood, i.e., have higher values in a fitness function.

Papers 3 and 4 are in finance. Naseem and Reesor assess the risk and reward of home borrowing for investment in the Canadian context, where it is possible to transform a non-tax deductible loan (personal mortgage) to tax deductible interest expenses by borrowing against home equity to make investments. Using a model that accommodates stochastically for changing interest rates and housing prices, the authors find that immediately borrowing from the increase in housing price not only decreases the expected mortgage payoff time, but also significantly increases the risk of taking a much longer time to pay off the mortgage. Trabelsi, He, He and Kusy examine the impact of the choice of cut-off points, sampling procedures, and business cycles on the forecasting accuracy of bankruptcy prediction models. They use a sample of firms from the Bankruptcy Research Database in the USA to evaluate the relative performance of the three most commonly used bankruptcy prediction models: Bayesian, Hazard, and Mixed Logit. They obtain that the choice of the cut-off point and sampling procedures affect indeed the rankings of the three models.

The fifth and sixth papers are applications in revenue management and energy market. In industries characterized by high fixed costs and low variable ones, e.g., the airline industry, adjusting supply and demand in view of maximizing revenues is a difficult task. Sharif Azadeh, Hosseinalifam and Savard evaluate the performance of a revenue management system when a non-parametric method for product choice probability estimation is chosen. The outcomes of this method are compared to the total expected revenue using synthetic data. De Matos, Sierra, Finardi, Decker and Milanezi introduce a stochastic model for energy marketing strategies of a small hydro plants (SHPs) in Brazil. The model is a multi-stage linear stochastic program, where the random variables are the energy future prices, the system hydro generation and the SHP generation in the portfolio. The authors use sampling strategies to identify a good solution. The paper also includes a case study to illustrate the type of results that can be obtained with the model consisting of a generator and a set of SHPs selling energy over periods between 6 and 24 months.

The last four papers are methodological contributions in different areas. Jaber and Sadeghigivi address some of the limitations in previous work on Wright's learning curve (WLC) in production. In particular, the authors allow for possible deterioration in knowledge between production cycles due to forgetting. Their results indicate that the performance function of the process has a convex form under certain conditions, and the performance of the system improves with faster learning in production and rework, frequent process restorations, and transfer of learning between cycles. Pakkar proposes a theoretical framework to assess the performance of decision making units (DMUs), which integrates the data envelopment analysis (DEA) and analytic hierarchy process (AHP) methodologies. To highlight the usefulness of the proposed approach, the author carries out a case study for assessing the financial performance of eight listed companies in the steel industry in China. 
In multi-stage stochastic optimization problems, the amount of stage-wise available information is crucial. While some authors deal with filtration distances, Timonina considers the concepts of nested distributions and their distances which allow keeping the setup purely distributional. She introduces the distance between stochastic process and a tree and generalizes the concept of nested distance two stochastic processes given by their continuous distributions. The author is making a step towards a new method for distribution quantization that is the most suitable for multi-stage stochastic optimization programs as it takes into account both the stochastic process and the stage-wise information. Finally, Sethuraman and Butenko consider the maximum ratio clique problem that aims at finding a maximal clique that has the largest ratio of benefit and cost weights associated with the clique's vertices. The authors establish the NP completeness of the decision version of the problem, and propose three solution methods. They report the results of numerical experiments with standard graph instances, as well as with real-life instances arising in finance and energy systems.

I would like to extend my warmest thanks to the reviewers who did an excellent job under very harsh time constraints. A special thank is also directed to the former editor-in-chief Berç Rustem for facilitating in many ways the process of planning and completing this issue. 